Recent data on the long-term outcome of antipsychotic treatment in first episode psychosis and schizophrenia: a critical perspective

Manuscript submitted to Psychological Medicine

Authors: Dr Joanna Moncrieff

Dr Sandra Steingard

Word count: 2,554 


\begin{abstract}
New studies of long-term outcomes claim to show that taking antipsychotics on a continuous and indefinite basis is the best approach for people diagnosed with a first episode of psychosis or schizophrenia. A 10-year follow-up of a trial of quetiapine maintenance, for example, found a higher proportion of people with a poor composite outcome in the group initially randomised to placebo. However, most people classified as showing poor outcome were rated as having a mild score on a single psychotic symptom; there were no differences in overall symptoms, positive or negative symptoms or level of functioning. Moreover, 16\% of participants did not have a follow-up interview and data from the end of the original trial were used instead. A study using a Finnish database suggested that mortality and readmission were higher in people who did not start long-term antipsychotic treatment or who discontinued it as compared to long-term continuous users. However, the analysis did not control for important confounders and is likely to reflect the fact that people who do not comply with treatment are at higher risk of death due to underlying health risks and behaviours. The analysis showed a slightly higher risk of readmission among non-users of antipsychotics compared to long-term users and a more substantial increased risk among people who discontinued treatment. However, follow-up ceased at the first readmission and therefore eventual, long-term outcome was not assessed. Speed of reduction and whether it was done with or without clinical support were also not distinguished.
\end{abstract}




\section{A critical analysis of recent data on the long-term outcome of antipsychotic treatment}

Long-term antipsychotic maintenance treatment has been the goal of treatment for schizophrenia for decades based on short-term studies that show the risk of relapse is elevated after abrupt antipsychotic discontinuation. Most psychiatrists assumed that by reducing risk of relapse one would be improving long-term outcome. However, some evidence suggests that long-term outcomes of neuroleptic discontinuation are not necessarily comparable to shortterm outcomes (Harrow et al. 2012; Moilanen et al. 2016; Morgan et al. 2014; Wils et al. 2017). Together with increasing evidence of neuroleptic-related adverse effects including structural brain changes, this has led some commentators to suggest that we should question the presumption of maintenance therapy for most people with psychotic disorders (Murray et al. 2016).

For people who have had a single episode of psychosis, current guidelines recommend that neuroleptics should be continued for a period of at least one to two years (National Institute for Health and Care Excellence, 2014), the idea being that if a person remains well during that time, it might then be safe to stop the drug. Many services now offer patients the option of reducing and stopping neuroleptic treatment after a variable period of maintenance treatment.

Two recent studies appear to challenge this tendency and the authors reassert the notion that long-term neuroleptic treatment should be the goal for the majority of people who experience psychosis, even after one episode. Recent reviews by eminent schizophrenia researchers reached the same conclusions (Correll et al. 2018; Goff et al. 2017). The new studies are important because they are some of the few that have gathered information over extended periods of time; therefore they merit close examination

\section{0-year follow-up of antipsychotic maintenance trial (Hui et al. 2018)}

One of these recent studies consists of a 10 year follow-up of participants who entered a randomised trial of quetiapine versus placebo maintenance treatment following a first episode of psychosis (Hui et al. 2018). The original trial, published in 2010, was conducted in Hong Kong and involved a sample of 178 patients (Chen et al. 2010). They were followed up for a 
year initially or until the first instance of relapse. Relapse was defined as the reappearance of one of 5 pre-specified Positive and Negative Syndrome Scale (PANSS) psychotic symptoms at threshold scores of between 3 (mild) and 5 (moderately severe) along with a Clinical Global Impressions-Severity score of 3 (mildly ill) or above and a Clinical Global ImpressionsImprovement score of 5 (minimally worse) or above. This definition was described by the authors as 'more liberal' than other studies and similar to some definitions of 'deterioration' (Chen et al. 2010) (P 3).

The initial results showed a risk of relapse of $79 \%$ in the group randomised to placebo, compared with $41 \%$ for the group allocated to quetiapine maintenance. Rates of hospitalisation were $16 \%$ and $6 \%$ respectively (Chen et al. 2010).

Ten year follow-up results compared the proportions of patients who had a 'poor' outcome, defined as a composite of persistent psychotic symptoms, being on clozapine, or death by suicide (Hui et al. 2018). Persistent psychotic symptoms were defined in the same way as they were for relapse in the initial analysis - in other words they could be judged as 'mild'. Researchers attempted to do a face to face follow-up interview with all the participants, but could only achieve this with 142 of the original sample. The analysis also included data for 28 patients $(16 \%)$ who were not interviewed at 10 years by using the last PANSS score obtained during the initial trial follow-up.

Using these definitions, a poor outcome was present in $21 \%$ of the group originally allocated to quetiapine maintenance and $39 \%$ of the placebo group ( $\mathrm{p}=0.012)$. However, looking at the components of the composite outcome in more detail reveals that most of those who were classified as having poor outcome were so defined by virtue of reaching threshold scores on the pre-specified psychotic symptoms. Most of these involved P1 (delusions) where the threshold criteria was 3 (mild). Inspecting other outcomes shows no difference in the overall PANSS score, the positive, negative and general subscales of the PANSS, rates of being in work and social functioning as measured by the Social and Occupational Functioning Assessment Scale (SOFAS). Slightly more people died by suicide or were prescribed clozapine in the group originally randomised to antipsychotic discontinuation, but numbers were small. It appears that the composite outcome may therefore have been constituted out of criteria selected post hoc on the basis of their differential distribution between the groups. 
Overall this study indicates that there was little difference in outcome between the groups 10 years after their enrolment in the antipsychotic discontinuation trial. This is not surprising given that the median duration of the original trial was only 145 days for the quetiapine group and 106 for the placebo group. Thus although it was described as a 12-month trial, in fact followup for most participants was much shorter, due to withdrawal or 'relapse'. Moreover, the inclusion of data from the original trial for some participants means the follow-up results do not reflect 10-year outcomes for all participants, and means the results presented are likely to mirror the results of the original trial.

\section{Retrospective database study (Tiihonen et al. 2018)}

The second study was a cohort study using routinely collected data from national registers concerning people who had a first admission for schizophrenia in Finland (Tiihonen et al. 2018). The main outcome was a composite variable called 'treatment failure' which consisted of a combination of rehospitalisation or death. Treatment failure was least common among continuous users of neuroleptics compared to non-users or people who discontinued. People who never started neuroleptics or who discontinued early were less likely to show treatment failure than those who took medication for longer periods and then stopped.

Again, the composite outcome, as well as lack of information about cause of death, makes the primary results difficult to interpret. Moreover, the observational nature of the data, and lack of matching or control for potential confounders makes it difficult to draw any causal inferences about the role of neuroleptic treatment.

It is recognised that observational data can be misleading because interventions are not allocated at random, even producing results that contradict those of randomised controlled trials (Freemantle et al. 2013). Careful matching or controlling potential confounders in the analysis may improve the reliability of the findings. In the present study, people were matched on the length of time they had been taking antipsychotics and age, sex and length of initial hospitalisation were adjusted for in the subsequent analysis. In a previous analysis of mortality data by the same group, the analysis also controlled for previous suicide attempt, cancer and ischaemic heart disease (Tiihonen et al. 2009). The authors were still criticised for not including other variables that might have important effects on mortality such as socioeconomic status, substance misuse, indicators of unhealthy lifestyle and detailed cardiovascular risk factors (De Hert et al. 2010). 
Evidence shows that people who are compliant with any treatment, including placebo, have better outcomes than those who are not across a range of conditions. People who stick to recommended treatment may, for example, be more physically or mentally healthy to begin with, have more stable lifestyles, be more likely to engage with other aspects of treatment and they may have more social support. In the Women's Health Initiative, for example, women who showed high compliance with placebo had lower rates of hip fractures, heart attacks, cancer death and death from all causes compared to those who showed poor placebo compliance (Curtis et al. 2011).

Clinicians' decisions to prescribe or to stop medication are also not random. In particular, clinicians may avoid prescribing a drug with known physical complications to someone who already has risk factors for those conditions. For example, evidence shows that clinicians prescribe a strongly weight-inducing drug like olanzapine to people with lower initial body weight than people to whom they prescribe other antipsychotics, such that olanzapine can be associated with lower body weight in observational data (Osborn et al. 2018). Reverse causality is also possible, since people who develop life-threatening conditions may have their prescription stopped subsequent to the onset of the illness but prior to actual death.

It is particularly difficult to interpret findings on mortality without a breakdown by cause of death. A similar registry data study conducted in Sweden showed that the majority of deaths, which were due to cardiovascular disease, showed a U-shaped relationship with antipsychotic exposure. People who were non-users had high rates of death, but there was a dose response relationship between antipsychotic exposure and death for those who had low, moderate and high exposure. The high mortality among non-users also held for cancer, suggesting that nonusers had higher underlying risk factors for cardiovascular mortality than users (Torniainen et al. 2015).

In the current study, figures presented in the supplemental information suggest that people who never used antipsychotics after discharge had the highest number of deaths, followed by those who discontinued antipsychotics within one year. Those who discontinued antipsychotics after longer periods did not, in fact, show higher rates of death compared with those who continued antipsychotics, although numbers of deaths for these groups were small. However, the figures exclude deaths that occurred after 24 hours of hospitalisation (to any hospital - personal communication). Since most people die in hospital, the data must considerably underestimate deaths in general, and possibly deaths in antipsychotic users in particular, as adherence is 
related to engagement with services which may make admission for a physical health event more likely.

As far as readmissions are concerned, the unmatched comparison (no matched group is provided for this comparison) found the risk of rehospitalisation was only slightly higher among non-users compared with continuous users of antipsychotic medication (Hazard Ratio 1.24 confidence interval 1.18 to 1.30 ).

In the comparison of 'matched' groups of continuous users and discontinuers, the risk of rehospitalisation increased with longer duration of treatment prior to discontinuation. The analysis consisted of a 'survival' analysis and follow-up ended at the time of rehospitalisation or when people switched antipsychotic status, i.e., when discontinuers started using antipsychotics again, or when users stopped taking them. In fact the numbers and proportions of people rehospitalised were comparable between continuous users and discontinuers, but length of follow-up was shorter with people who discontinued antipsychotics being followed up for a median of between 122 and 134 days compared to 376 for continuous users. What the analysis shows, therefore, is that readmissions occurred earlier in people who had discontinued antipsychotics, but not necessarily that they were more common. The result is consistent with other evidence showing that stopping antipsychotics brings forward risk of relapse but may not influence risk in the long-run (Wunderink et al. 2013).

The focus on short-term events may explain the discrepancy between the present study and the findings of long-term cohort studies, which suggest that people who avoid long-term continuous antipsychotic treatment have better outcomes, including lower rates of relapse and higher levels of functioning and recovery, compared to continuous users (Harrow et al. 2012; Moilanen et al. 2016; Morgan et al. 2014; Wils et al. 2017). These studies are also subject to the biases that arise because treatment is not randomly allocated, but they do provide face to face follow-up over long periods with all participants who can be located.

The benefits that non-users of antipsychotics showed in one cohort study that tracked progress every few years only started to become apparent at the four-year follow-up point (Harrow et al. 2012). Although the present study is reported as a 20-year follow-up, this is the maximum duration, but the median follow-up was only 17- 19 weeks for those who discontinued antipsychotics, 54 weeks for continuous users and 58 weeks for non-users (408 days).

\section{Reverse causality may also operate in relation to the outcome of rehospitalisation in circumstances when a deterioration in mental state leads to discontinuation of}


antipsychotics that pre-dates hospitalisation. This is especially likely given that most instances of antipsychotic discontinuation in this study are likely to have been unsupervised.

Given the caveats about observational studies, the present study may indicate an increased risk of readmission to hospital following antipsychotic discontinuation, but it is not a study of a controlled and gradual reduction of antipsychotics done with professional support. It also provides no data on other outcomes such as quality of life and social functioning or on the burden of drug-related adverse effects that patients can suffer.

\section{Conclusion}

Studies that trace the long-term outcome of psychosis and its relation to treatment strategies are important but not easy to achieve. Experts defending the long-term use of neuroleptics acknowledge there is little robust evidence of long-term outcomes and place much emphasis on the observational analyses of mortality data such as the Finnish study described above (Correll et al. 2018; Goff et al. 2017). We suggest there is an insufficient appreciation of the potentially misleading nature of such analyses. The follow-up data from the randomised trial of quetiapine maintenance is important, therefore, but the main composite outcome variable is difficult to interpret and other outcomes showed no difference between groups. Studies suggesting better outcomes for people who discontinue antipsychotic treatment also have limitations. Cohort studies are confounded by likely differences in severity and other factors associated with outcome. The seven year follow-up of participants from a randomised controlled trial of antipsychotic reduction (Wunderink et al. 2013) has been criticised for not controlling treatment adherence throughout follow-up and for high rates of crossover (Undurraga et al. 2014; Pies 2016). These points do not detract from the ability of the study to evaluate the long-term effects of an initial, pragmatic antipsychotic reduction programme, however, but suggest that the study does not reflect a comparison of continuous antipsychotic treatment compared with no treatment over the whole of the seven year follow-up period. Nevertheless, the small size of the study means that it needs replicating. Ultimately, there is a need for further long-term follow up of randomised cohorts to determine the full risks and benefits of continuous neuroleptic treatment compared to other treatment strategies over the long-term. 
Data from the Finnish database study also reveal that over $43 \%$ of individuals with a first diagnosis of schizophrenia never start neuroleptics and by one year, almost $57 \%$ of those who do have stopped. This indicates that many people do not want to take these drugs and regardless of current data, professionals still need to engage with them in ways that might help them navigate the world with less risk to their well-being and safety. It is also interesting that while the rate of rehospitalisation was higher among people who discontinued antipsychotics (36\%), it was not substantially lower among the 'matched' group of continuous users (27\%). A reasonable person might accept a higher (and not inevitable) risk of recurrence of psychosis, in the full knowledge of what that might entail, and decide to stop medication, as a way to minimize the long-term risks of negative outcomes associated with staying on the drugs. As professionals, it is important to help people to minimise the risks of this approach. Although there is little research on how to achieve this, it seems sensible to recommend a gradual process of reduction that might, at least, allow the individual and treatment team time to recognize early signs of relapse and intervene accordingly. 
Reference List

Chen, E. Y., Hui, C. L., Lam, M. M., Chiu, C. P., Law, C. W., Chung, D. W., Tso, S., Pang, E. P., Chan, K. T., Wong, Y. C., Mo, F. Y., Chan, K. P., Yao, T. J., Hung, S. F. \& Honer, W. G. (2010). Maintenance treatment with quetiapine versus discontinuation after one year of treatment in patients with remitted first episode psychosis: randomised controlled trial. British Medical Journal 341, c4024 Aug 19;341:c4024. doi: 10.1136/bmj.c4024.

Correll, C. U., Rubio, J. M. \& Kane, J. M. (2018). What is the risk-benefit ratio of long-term antipsychotic treatment in people with schizophrenia? World Psychiatry 17, 149-160.

Curtis, J. R., Larson, J. C., Delzell, E., Brookhart, M. A., Cadarette, S. M., Chlebowski, R., Judd, S., Safford, M., Solomon, D. H. \& Lacroix, A. Z. (2011). Placebo adherence, clinical outcomes, and mortality in the women's health initiative randomized hormone therapy trials. Medical Care 49, 427-435.

De Hert, M., Correll, C. U. \& Cohen, D. (2010). Do antipsychotic medications reduce or increase mortality in schizophrenia? A critical appraisal of the FIN-11 study. Schizophrenia Research. 117, 68-74.

Freemantle, N., Marston, L., Walters, K., Wood, J., Reynolds, M. R. \& Petersen, I. (2013). Making inferences on treatment effects from real world data: propensity scores, confounding by indication, and other perils for the unwary in observational research. British Medical Journal 347, f6409. doi: 10.1136/bmj.f6409 .

Goff, D. C., Falkai, P., Fleischhacker, W. W., Girgis, R. R., Kahn, R. M., Uchida, H., Zhao, J. \& Lieberman, J. A. (2017). The Long-Term Effects of Antipsychotic Medication on Clinical Course in Schizophrenia. American Journal of Psychiatry, 174, 840-849.

Harrow, M., Jobe, T. H. \& Faull, R. N. (2012). Do all schizophrenia patients need antipsychotic treatment continuously throughout their lifetime? A 20-year longitudinal study. Psychological Medicine 42, 2145-55.

Hui, C. L. M., Honer, W. G., Lee, E. H. M., Chang, W. C., Chan, S. K. W., Chen, E. S. M., Pang, E. P. F., Lui, S. S. Y., Chung, D. W. S., Yeung, W. S., Ng, R. M. K., Lo, W. T. L., Jones, P. B., Sham, P. \& Chen, E. Y. H. (2018). Long-term effects of discontinuation from antipsychotic maintenance following first-episode schizophrenia and related disorders: a 10 year follow-up of a randomised, double-blind trial. Lancet Psychiatry 5, 432-442.

Moilanen, J. M., Haapea, M., Jaaskelainen, E., Veijola, J. M., Isohanni, M. K., Koponen, H. J. \& Miettunene, J. (2016). Long-term antipsychotic use and its association with 
outcomes in schizophrenia: the Northern Finland birth cohort 1966. European Psychiatry 36, 7-14.

Morgan, C., Lappin, J., Heslin, M., Donoghue, K., Lomas, B., Reininghaus, U., Onyejiaka, A., Croudace, T., Jones, P. B., Murray, R. M., Fearon, P., Doody, G. A. \& Dazzan, P. (2014). Reappraising the long-term course and outcome of psychotic disorders: the AESOP-10 study. Psychological Medicine 44, 2713-2726.

Murray, R. M., Quattrone, D., Natesan, S., van Os, J., Nordentoft, M., Howes, O., Di Forti, M. \& Taylor, D. (2016). Should psychiatrists be more cautious about the longterm use of antipsychotics? British Journal of Psychiatry 209, 361-365.

National Institute for Health and Care Excellence (NICE) (2014). Psychosis and schizophrenia in adults: treatment and management. NICE: London.

Osborn, D. P., Petersen, I., Beckley, N., Walters, K., Nazareth, I. \& Hayes, J. (2018). Weight change over two years in people prescribed olanzapine, quetiapine and risperidone in UK primary care: Cohort study in THIN, a UK primary care database. Journal of Psychopharmacol. Jun 1:269881118780011. doi: 10.1177/0269881118780011. [Epub ahead of print].

Pies, R. (2016). Long-term Antipsychotic Treatment: Effective and Often Necessary, with Caveats. Psychiatric Times 33.

Tiihonen, J., Lonnqvist, J., Wahlbeck, K., Klaukka, T., Niskanen, L., Tanskanen, A. \& Haukka, J. (2009). 11-year follow-up of mortality in patients with schizophrenia: a population-based cohort study (FIN11 study). Lancet 374, 620-627.

Tiihonen, J., Tanskanen, A. \& Taipale, H. (2018). 20-Year Nationwide Follow-Up Study on Discontinuation of Antipsychotic Treatment in First-Episode Schizophrenia. American Journal of Psychiatry, Apr 6:appiajp201817091001. doi: 10.1176/appi.ajp.2018.17091001. [Epub ahead of print] .

Torniainen, M., Mittendorfer-Rutz, E., Tanskanen, A., Bjorkenstam, C., Suvisaari, J., Alexanderson, K. \& Tiihonen, J. (2015). Antipsychotic treatment and mortality in schizophrenia. Schizophrenia Bulletin 41, 656-663.

Undurraga, J., Murru, A. \& Vieta, E. (2014). Early medication discontinuation on longterm recovery outcome in first-episode psychosis. JAMA Psychiatry 71, 206-207.

Wils, R. S., Gotfredsen, D. R., Hjorthoj, C., Austin, S. F., Albert, N., Secher, R. G., Thorup, A. A., Mors, O. \& Nordentoft, M. (2017). Antipsychotic medication and remission of psychotic symptoms 10 years after a first-episode psychosis. Schizophrenia Research 182, 42-48.

Wunderink, L., Nieboer, R. M., Wiersma, D., Sytema, S. \& Nienhuis, F. J. (2013). Recovery in Remitted First-Episode Psychosis at 7 Years of Follow-up of an Early Dose Reduction/Discontinuation or Maintenance Treatment Strategy: Long-term Follow-up of a 2-Year Randomized Clinical Trial. Journal of the American Medical Association: Psychiatry, 70, 913-20. 
